\title{
VESGEN 2D: Automated, User-Interactive Software for Vascular Quantification and Mapping of Angiogenic and Lymphangiogenic Trees and Networks
}

\author{
Mary B. Vickerman ${ }^{1}$, Patricia A. Keith ${ }^{1}$, Terri L. McKay ${ }^{1}$, Dan J. Gedeon ${ }^{1}$, Michiko \\ Watanabe $^{2,3}$, Monica Montano ${ }^{4}$, Ganga Karunamuni ${ }^{2}$, Peter K. Kaiser ${ }^{5}$, Jonathan E. Sears ${ }^{5}$, \\ Quteba Ebrahem $^{5}$, Daniela Ribita ${ }^{1}$, Alan G. Hylton ${ }^{1}$, and Patricia Parsons-Wingerter ${ }^{1,6}$ \\ ${ }^{1}$ NASA Glenn Research Center, Cleveland, OH 44135 \\ ${ }^{2}$ Department of Anatomy, Case Western Reserve University School of Medicine, Cleveland, $\mathrm{OH}$ \\ 44106 \\ ${ }^{3}$ Department of Pediatric Cardiology, Case Western Reserve University School of Medicine, \\ Cleveland, $\mathrm{OH} 44106$ \\ ${ }^{4}$ Department of Phamacology, Case Western Reserve University School of Medicine, Cleveland, \\ $\mathrm{OH} 44106$ \\ ${ }^{5}$ Cole Eye Institute, Lerner Research Institute, Cleveland Clinic Foundation, Cleveland, OH 44195 \\ ${ }^{6}$ Department of Cell Biology, Lerner Research Institute, Cleveland Clinic Foundation, Cleveland, \\ $\mathrm{OH} 44195$
}

\author{
Abstract \\ Quantification of microvascular remodeling as a meaningful discovery tool requires mapping and \\ measurement of site-specific changes within vascular trees and networks. Vessel density and other \\ critical vascular parameters are often modulated by molecular regulators as determined by local \\ vascular architecture. For example, enlargement of vessel diameter by vascular endothelial growth \\ factor (VEGF) is restricted to specific generations of vessel branching (Microvascular Research \\ 72(3):91, 2006). The averaging of vessel diameter over many successively smaller generations is \\ therefore not particularly useful. The newly automated, user-interactive software VESGEN \\ (VESsel GENeration Analysis) quantifies major vessel parameters within two-dimensional (2D) \\ vascular trees, networks, and tree-network composites. This report reviews application of \\ VESGEN 2D to angiogenic and lymphangiogenic tissues that includes the human and murine \\ retina, embryonic coronary vessels, and avian chorioallantoic membrane (CAM). Software output \\ includes colorized image maps with quantification of local vessel diameter, fractal dimension, \\ tortuosity and avascular spacing. The density of parameters such as vessel area, length, number \\ and branch point are quantified according to site-specific generational branching within vascular \\ trees. The sole user input requirement is a binary (black/white) vascular image. Future applications \\ of VESGEN will include analysis of 3D vascular architecture and bioinformatic dimensions such \\ as blood flow and receptor localization. Branching analysis by VESGEN has demonstrated that \\ numerous regulators including $\mathrm{VEGF}_{165}$, basic fibroblast growth factor (bFGF), transforming \\ growth factor $\beta-1$ (TGF $\beta-1)$, angiostatin and the clinical steroid triamcinolone acetonide induce \\ 'fingerprint' or 'signature' changes in vascular patterning that provide unique readouts of \\ dominant molecular signaling.
}




\section{Keywords}

angiogenesis; lymphangiogenesis; VESGEN; retina; neovascularization; NPDR; coronary vascular development; vascular complexity; VEGF; fractal; chorioallantoic membrane; CAM; quail; avian; triamcinolone acetonide

\section{INTRODUCTION}

Microvascular remodeling is now widely acknowledged as fundamental to normal physiological processes that include embryonic development, reproductive biology and healthy wound-healing, and the progressive pathologies of neovascular diseases such as cancer, diabetes and heart disease (Folkman, 2007). However, angiogenesis, lymphangiogenesis and other microvascular remodeling processes are difficult to map and quantify because of the morphological complexity of branching vascular trees and their associated capillary networks. Furthermore, the architecture of a vascular tree or network is locally adapted to specific needs of the host tissue or organ. In general, blood and lymphatic vascular structures can be classified as (1) heterogeneous, asymmetric trees of vessels that branch and taper; (2) relatively homogeneous, symmetric networks or plexuses, and (3) treenetwork composites. Mature vascular trees typically develop from immature, capillary-like vasculogenic networks. Within a mature organ or tissue, capillary networks are necessarily continuous with their arterial and venous trees.

The computer software VESGEN (VESsel GENeration Analysis) maps and quantifies major parameters of angiogenesis and lymphangiogenesis in vascular trees and networks. This review describes results generated by the newly automated VESGEN 2D software (version 1.0) that analyzes a number of two-dimensional (2D) research and clinical applications. VESGEN 2D will be released in the near future as an easily installed software plug-in for the widely utilized image processing program ImageJ. Output of VESGEN 2D includes vessel diameter, tortuosity, fractal dimension, densities of vessel area, length, number and branch point, and a variety of useful image mappings. The vascular tree is decomposed into its branching generations so that cytokine- or therapeutic-regulated modifications can be quantified according to branch-specific vessel location. For example, quantifying an average vessel diameter over many branching generations is not a particularly useful result. For capillary, vasculogenic and lymphatic networks, fundamental relationships between vessel morphology and avascular spaces are quantified.

Results generated by the VESGEN 2D 1.0 are reviewed as vascular trees, networks, and tree-network composites (the three morphological options available in VESGEN 2D). Applications include progression of neovascular disease in clinical images of the human retina, effects of the stimulator vascular endothelial growth factor-165 $\left(\mathrm{VEGF}_{165}\right)$ and inhibitor triamcinolone acetonide (TA) on vascular or lymphatic trees and networks in the developing mouse retina and quail chorioallantoic membrane (CAM), and vascular networkto-tree transitions for developing coronary vessels. We use the quail CAM as a convenient experimental and analytical testbed for development of new quantification methods (Parsons-Wingerter et al., 1998; Parsons-Wingerter et al., 2000a; Parsons-Wingerter et al., 2000b; Parsons-Wingerter et al., 2006a; Parsons-Wingerter et al., 2006b; McKay et al., 2008) that have been extended successfully, for example, to a novel analysis of disease progression in the human retina (Avakian et al., 2002). 


\section{BACKGROUND}

\section{Models of Angiogenesis and Lymphangiogenesis}

Vascular Trees and Lymphatic Networks in the Avian CAM-Assay and microscopic imaging of the CAM model are described elsewhere (Parsons-Wingerter et al., 1998; Parsons-Wingerter et al., 2000a; Parsons-Wingerter et al., 2000b; Lazarovici et al., 2006; Parsons-Wingerter et al., 2006a; Parsons-Wingerter et al., 2006b). We use the quail CAM because it is small (approximately one-fifth the size of a chicken CAM). Quantification is convenient (Fig. 1) because the rapidly growing vascular trees and associated lymphatic networks (Fig. 6) are quasi-2D and embedded within transparent CAM tissue. Our development of quantification in the CAM has focused primarily on describing angiogenic changes in the vascular tree, rather than the capillary network, because of our particular interest in branching phenomena.

Briefly, fertilized eggs of Japanese quail (Coturnix coturnix japonica) were incubated at $37.6 \pm 0.2{ }^{\circ} \mathrm{C}$, cracked at embryonic day three (E3), and cultured further in Petri dishes. At $\mathrm{E} 7$, solutions containing the perturbant $\left(\mathrm{TA}\right.$ or $\mathrm{VEGF}_{165}$ ) were applied topically to the entire surface of the CAM, resulting in uniform stimulation or inhibition of all CAM vasculature (due to flatness of CAM surface). After incubation for an additional 24 hours (to E8) or 48 hours (to E9), CAMs were aldehyde-fixed and dissected. For bright-field microscopy of CAM vasculature, (Fig. 1), the arterial tree was isolated and distinguished from the venous tree by a well-established artifact of CAM fixation, in which blood is retained as the optical contrast marker within arteries, but not within veins, following fixation at high concentrations of paraformaldehyde and glutaraldehyde (Parsons-Wingerter et al., 1998). For confocal microscopy (Fig. 6), both blood and lymphatic vessels were identified positively by fluorescence immunohistochemistry using an antibody recognizing VEGF receptor-2 (VEGFR-2) and the QH-1 monoclonal antibody that recognizes quail hematopoietic precursors and blood and lymphatic vasculature (Parsons-Wingerter et al., 2006a). Blood and lymphatic vessels were distinguished from each other by positive immunological labeling of the blood vessels for $\alpha$ smooth muscle actin ( $\alpha$ SMA) and of the lymphatic vessels, for prospero-related Homeobox 1 (Prox1).

Vascular Trees in the Human Retina-Grayscale digital images $(2392 \times 2048$ pixels $)$ of the human retina were obtained by $50^{\circ}$ clinical fluorescein angiography (FA) from patients diagnosed with nonproliferative diabetic retinopathy (NPDR) according to standards of the Early Treatment Diabetic Retinopathy Study (ETDRS). Clinical FA is performed by injecting fluorescein into the vasculature, followed by fluorescence imaging of retinal vascular filling. FA is an important tool in the diagnosis and treatment of DR, because vessels and other important vascular anomalies such as microaneurysms are visualized with greater resolution and clarity than in color fundus photography. Within each angiogram, the arterial and venous trees were extracted as overlapping binary vascular patterns by semiautomatic computer processing (Avakian et al., 2002) and then separated according to: 1) staging of arteriovenous filling by the injected fluorescein tracer, in which arteries are filled first before the veins; 2) characteristic arterial and venous morphology, and 3) basic principles of vascular tree connectivity, branching and tapering. For example, arterial and venous trees tend to originate from the optic nerve in pairs, and arterial vessels are more tortuous and of smaller diameter than venous vessels. Once a vascular tree has been identified as arterial or venous at its origin according to vascular morphology and fluorescein filling, the tree can be followed from its origin to its termination at the smallest generations according to principles of vessel connectivity, bifurcational branching and tapering (the basic morphological characteristics of a mature vascular tree). Following automatic analysis by VESGEN 2D of the four retinal images (two arterial trees and two 
venous trees), 5 vessels out of 1760 total vessels appeared incorrectly assigned to their respective branching generations, and were therefore corrected by user-active VESGEN 2D interventions.

Vascular Networks in the Mouse Retina-Vascular networks were identified by fluorescence microscopy for retinal flat mounts obtained from mice at postnatal day 15 (P15), when the retina and its blood vessels are still developing. Retinas were carefully dissected away from eyes following fixation in $4 \%$ paraformaldehyde. For one study, retinal vasculature was visualized in transgenic mice by expression of green fluorescence protein (GFP) driven by the Tie2/Tek promoter (Jackson Laboratories). In another study, mice were injected subcutaneously in the neck with $4 \mathrm{mg}$ of TA or PBS vehicle at P4 and P7, and vessels labeled by injection of lectin-conjugated fluorescein into the heart prior to sacrifice at P15.

Network-Tree Transitions of Embryonic Coronary Vessels-Developing coronary vessels in mouse and chicken embryonic models from various stages of heart development were imaged by brightfield, fluorescence or fluorescence confocal microscopy, and transformed into binary vascular patterns for VESGEN 2D network or tree analysis by semiautomatic computer processing as described above.

\section{VESGEN 2D Software}

Description-VESGEN 2D is an automated microvascular mapping and quantification software that provides an interactive, flexible, menu-driven user interface (UI) to accommodate the evolving needs of researchers grappling with the complexities of in vivo vascular morphological analysis for angiogenesis and lymphangiogenesis applications. VESGEN 2D is a mature pre-release software program developed by the John Glenn NASA Research Center that will be publicly available in the near future following development of user documentation. Written in Java with over 11,000 lines of code, VESGEN 2D operates as an easily installable plug-in to the free, downloadable software ImageJ (National Institutes of Health). Initial results for VESGEN 2D were generated by a semi-automatic Matlab ${ }^{\mathrm{R}}$-based computer code prototype (Parsons-Wingerter et al., 1998; Parsons-Wingerter et al., 2000a; Parsons-Wingerter et al., 2000b; Parsons-Wingerter et al., 2006b) and a distance mapping (DM) algorithm (Parsons-Wingerter et al., 2006a). These and other new advanced, automated capabilities have been incorporated into the current version of the VESGEN 2D software (see also McKay et al., 2008).

The sole VESGEN 2D input requirement is a user-provided vascular binary image, for which one of the three analysis options can be selected: (1) Vascular Tree, (2) Vascular Network, or (3) Tree-Network Composite. Output data include images processed into various vascular maps (for example, see Fig. 1D and Fig. 2B, C), overall image measurements, and measurements specified for individual vascular branching generations. To analyze the input image, the user selects the desired analytical option from the UI, further selects the desired series of output images, and then performs custom measurements on the image collection. The basic three analytical options, their capabilities, and output are further described below.

Capabilities-VESGEN 2D analyzes a binary vascular image according to the userselected tree, network or composite tree-network option. Parameters are reported in pixel units and optionally in physical units if a microscope calibration factor is provided by the user. Image processing algorithms include those in the standard toolset provided by ImageJ, as well as customized algorithms written for, and unique to, VESGEN 2D. For example, several methods of automatic ROI detection are available, such as an ROI method that finds the midpoint between a selected vessel region (such as the largest vascular tree) and other 
non-selected vessels. In order to analyze the successive branching generations of vascular trees, highly specialized algorithms determine the boundaries of branches and assign branches to the appropriate branching generation.

The user may interact with VESGEN 2D for optimization of the output analysis if desired. For example, after a vascular tree has been analyzed and mapped into all generations of vessel branching (which in our research have varied typically from six to thirteen branching generations), the user can re-combine the generational results into specified classes of branching, such as small, medium and large branching generations (Parsons-Wingerter et al., 2006b; McKay et al., 2008). The vascular region of interest (ROI) is automatically determined by VESGEN 2D or provided by the user. Output results include vascular maps illustrating generational assignments for vascular trees, distance maps displaying local vessel diameter, the ROI used to calculate vessel density parameters (described below), and output measurements provided as a one-page summary and detailed multi-page measurement documentation (for example, see Fig. 1D and Fig. 2B, C).

Vascular Trees-Vascular trees are defined as highly branching, asymmetric, nonhomogeneous, structures comprised of tapering vessels. When the Vascular Tree option is selected, vessel parameters are measured by VESGEN 2D in a region of interest (ROI) within the vascular input image. Options for the ROI are: (1) the entire image, (2) the major vascular tree within the image (Fig. 1), or (3) a vascular tree selected by the user. Vascular tree parameters include vessel diameter $\left(D_{v}\right)$, vessel tortuosity $\left(T_{v}\right)$, fractal dimension $\left(D_{f}\right)$, vessel area density $\left(A_{v}\right)$, vessel length density $\left(L_{v}\right)$, vessel number density $\left(N_{v}\right)$, and vessel branch point density $\left(\mathrm{Br}_{\mathrm{v}}\right)$. For example, $\mathrm{A}_{\mathrm{v}}$ is the vessel area divided by the area of the ROI. $\mathrm{D}_{\mathrm{f}}$ is measured by the box-counting algorithm. Fractal analysis is a relatively new, non-Euclidean geometric tool (Mandelbrot, 1983) that is useful for quantifying complex space-filling objects such as branching vascular trees, particularly when the object demonstrates the geometric property of self-similarity (i.e., repetition of a pattern such as vascular bifurcational branching at increasingly smaller length scales).

Averaging of many parameters of a vascular tree such as vessel diameter or vessel length is not a highly meaningful analysis when these parameters vary so greatly throughout a branching vascular tree. Therefore VESGEN 2D measures many of these parameters according to their site-specific location within the vascular tree. For example, $\mathrm{D}_{\mathrm{v} 1-3}$ denotes vessel diameter $D_{v}$ with respect to branching generations G1-G3, and $N_{v 6}$ is the vessel number density $\mathrm{N}_{\mathrm{v}}$ for branching generation G6.

To accomplish the vessel segmentation (i.e., assignment of branching generations), vessels of the major vascular tree and throughout the entire image are segmented (decomposed) into successively smaller branching generations $\left(\mathrm{G}_{1}, \mathrm{G}_{2}, \ldots \mathrm{G}_{\mathrm{X}}\right)$ according to a proportional decrease of vessel diameter. The largest vessel is designated as $\mathrm{G}_{1}$. At a symmetric vessel branching or offspring (daughter) bifurcation, blood flows most efficiently when the diameters of the two offspring vessels are $71 \%$ (square root of 2) of the parent vessel diameter. Therefore vessels are assigned to the next offspring branching generation such as $\mathrm{G}_{2}$ from $\mathrm{G}_{1}$ when diameters of the offspring vessels are $71 \%$ of the parent vessel. This symmetric bifurcational offspring arrangement would dictate the branching architecture of a geometrically perfect branching tree (if symmetric branching angles and lengths were also included). However, cardiovascular and other biological trees are more variable in their construction. Hence VESGEN 2D assigns a vessel to its branching generation by applying a tolerance factor to the $71 \%$ rule for relatively symmetric offspring vessel bifurcations. The VESGEN 2D default tolerance factor of $15 \%$ was used in this study (this tolerance factor is user-adjustable), so that the overriding criterion is $71 \% \pm 15 \%$ at a relatively symmetric offspring vessel bifurcation. 
Another analysis consideration is that the most frequent branching event within a vascular tree is the asymmetric offshoot of a much smaller vessel from a larger vessel (Fig. 1), which presumably supports the uniform, efficient delivery of blood throughout the tissue. Due to these smaller asymmetric offshoot vessels, larger vessels taper throughout the tree, although tapering may also support the mechanics of blood flow. Thus occasionally a parent vessel can become an offspring vessel when its local diameter reaches $71 \%-15 \%$, or $56 \%$ of the average vessel diameter (Fig.1).

Vascular Networks-Vascular networks are relatively symmetric, homogeneous, nonbranching structures characterized by enclosed avascular spaces (i.e., holes or lacunae; see Fig. 2). Vascular trees often develop from vasculogenic capillary networks, and mature capillaries are typically organized as networks. A basic principle of vascular network analysis is that the fractional areas of network vessels and avascular spaces must sum to 1, when normalized by the total ROI area. The two extremes of thin capillary vessels with large avascular spaces and thick 'presinusoidal' capillary vessels with small avascular spaces were contrasted in an interesting study of these geometric relationships (LaRue et al., 2003). Upon selection of the Vascular Network option, VESGEN 2D analyzes the vascular binary input image for parameters that include $\mathrm{D}_{\mathrm{f}}$, other relevant vascular parameters such as $A_{v}, L_{v}$, and $B r_{v}$, histogram data of vessel diameters (sampled along the vessel skeleton), and number and areas of avascular spaces.

Tree-Network Composites-Vascular tree-network composites are structures that are geometrically intermediate between trees and networks (Fig. 8), and often represent a transitional state of development from an immature network to a more mature tree. When the Vascular Tree-Network Composite option is selected, vessel parameters are generated as for the Vascular Tree and Vascular Network options described above. VESGEN 2D quantifies the vascular binary input image for parameters that include overall image results as well as generation-based measurements and avascular spaces measurements. In the future, we will develop further capabilities for the Vascular Network and Vascular Tree-Network Composite options.

\section{VESGEN 2D RESULTS}

Quantification and vascular mapping by VESGEN 2D are reviewed for several experimental and clinical models in vivo. Applications include progression of vascular disease in the human retina from ophthalmic clinical images; effects of angiogenic and lymphangiogenic regulators in the avian CAM and developing murine retina, and development of murine and avian embryonic coronary vessels. Results are presented according to the three VESGEN 2D user options of Vascular Trees, Vascular Networks, and Vascular Tree-Network Composites.

\section{Disease Progression in Vascular Trees of Human Retina}

Major sources of blindness in diabetic retinopathy and other retinal vascular diseases are macular ischemia and retinal neovascularization, which impairs retinal function and can obscure retinal vision through hemorrhage and eventually traction retinal detachment. The presence of neovascularization defines late-stage diabetic retinal disease, which is termed proliferative diabetic retinopathy (PDR). Early-stage, non-proliferative disease (NPDR) is characterized by vascular dropout and secondary effects of adverse vascular remodeling with no neovascularization (Avakian et al., 2002), in which stages of NPDR are defined as mild, moderate, severe and very severe. As a preliminary methodological study, we applied VESGEN 2D analysis to the progression of NPDR. For the blinded analysis, two representative clinical FA images (Fig. 3A, C) were analyzed by VESGEN 2D (Fig. 4) from 
patients with mild/moderate NPDR and severe NPDR. All results of this methodological analysis would require confirmation by much larger research and clinical studies.

Inspection of the extracted arterial and venous trees of the mild/moderate versus severe NPDR FA images (Fig. 3B, D) clearly suggests a decrease in vessel density, which could derive from progressive vessel dropout. By VESGEN 2D quantification of eight to nine branching generations (G1 to G8 or G9, Fig. 4), parameters that include the fractal dimension $\left(\mathrm{D}_{\mathrm{f}}\right)$ and vessel densities of area $\left(\mathrm{A}_{\mathrm{v}}\right)$, length $\left(\mathrm{L}_{\mathrm{V}}\right)$, number $\left(\mathrm{N}_{\mathrm{v}}\right)$ and branchpoint $\left(\mathrm{Br}_{\mathrm{v}}\right)$ confirm that vessel density decreased significantly in the severe NPDR FA image compared to the mild/moderate NPDR FA image. In arterial trees, $\mathrm{D}_{\mathrm{f}}$ (a very sensitive indicator) decreased from 1.39 to 1.27 , and $\mathrm{A}_{\mathrm{v} 1-9}, \mathrm{~L}_{\mathrm{v} 1-9}, \mathrm{Br}_{\mathrm{v} 1-9}$ and $\mathrm{N}_{\mathrm{v} 1-9}$ decreased by $30 \%, 51 \%$, and $78 \%$ and $74 \%$, respectively. Decreases in venous density were less marked.

\section{Anti-Angiogenesis Effects of a Clinical Steroid in Vascular Trees of Avian CAM}

The anti-inflammatory corticosteroid TA is an important clinical drug that is injected into the body, often in targeted sites such as the eye, to counteract progression of numerous pathologies, including neovascularization and edema in diabetic retinopathy (Moshfeghi et al., 2005; Brasil et al., 2007; Galor et al., 2007). For increased understanding of how TA effects vascular morphology, TA was applied topically to the angiogenic vasculature of the quail CAM, and results were analyzed by VESGEN 2D (Fig. 5). Each representative image displays the major arterial tree with its ROI (in black) as determined by VESGEN 2D, for which vessel diameter $\left(D_{\mathrm{v}}\right)$ and the density of key vascular parameters that include vessel number density $\left(\mathrm{N}_{\mathrm{v}}\right)$, vessel branch point density $\left(\mathrm{Br}_{\mathrm{v}}\right)$, vessel length density $\left(\mathrm{L}_{\mathrm{v}}\right)$, and vessel area density $\left(A_{v}\right)$, were measured.

By $N_{v}$ and $L_{v}$ (Fig. 5), vessel density decreased significantly for the smallest vessels of $G_{7}{ }^{-}$ $\mathrm{G}_{10}$, but not for large and medium-sized vessels of generations $\mathrm{G}_{1}-\mathrm{G}_{2}, \mathrm{G}_{3}-\mathrm{G}_{4}$ and $\mathrm{G}_{5}-\mathrm{G}_{6}$. For example, $\mathrm{N}_{\mathrm{v} 7-10}$ decreased significantly from $474 \mathrm{~cm}^{-2}$ in the control to $302 \mathrm{~cm}^{-2}$ at 8 ng TA/CAM, whereas $\mathrm{N}_{\mathrm{v} 1-2}, \mathrm{~N}_{\mathrm{v} 3-4}$ and $\mathrm{N}_{\mathrm{v} 5-6}$ remained relatively constant following TA treatment. $\mathrm{N}_{\mathrm{v} 1-2}$ was $5.04 \mathrm{~cm}^{-2}$ in control compared to $5.15 \mathrm{~cm}^{-2}$ at $8 \mathrm{ng}$ TA/CAM. Small but consistent decreases in vessel diameter $\left(\mathrm{D}_{\mathrm{v}}\right)$ were induced by TA throughout the vascular tree. $D_{\mathrm{v} 7-10}$ decreased from $28.1 \pm 1.0 \mu \mathrm{m}$ in control to $25.5 \pm 0.4 \mu \mathrm{m}$ at $8 \mathrm{ng}$ TA/ CAM, $\mathrm{D}_{\mathrm{v} 5-6}$ from $61.1 \pm 3.1 \mu \mathrm{m}$ to $53.0 \pm 3.1 \mu \mathrm{m}, \mathrm{D}_{\mathrm{v} 3-4}$ from $118.1 \pm 6.0 \mu \mathrm{m}$ to $101.6 \pm$ $5.8 \mu \mathrm{m}$, and $\mathrm{D}_{\mathrm{v} 1-2}$ from $228.4 \pm 12.1 \mu \mathrm{m}$ to $199.0 \pm 11.2 \mu \mathrm{m}$. Vessel tortuosity as measured by $T_{V}$ was unaffected. Therefore TA selectively decreased angiogenesis only in smaller vessels (as measured by several confirming parameters for vessel density), but in contrast, decreased vessel diameter throughout all branching generations of the vascular tree. Detailed results of a large, comprehensive dose-response study of TA in the CAM model are described elsewhere (McKay et al., 2008).

\section{TA Regulation of Vascular Networks in Mouse Retina}

The early postnatal mouse retina is a well-established in vivo model for examining retinal vascular development and investigating retinal neovascular pathologies such as infant retinopathy of prematurity (ROP). At P15, for example, the mouse retina displays peripheral vasculogenic capillary networks that will quickly remodel into mature vascular trees (Benjamin et al., 1998; Gerhardt et al., 2003). In the experimental model described in Fig. 6, Fig. $4 \mathrm{mg}$ of anti-angiogenic clinical steroid TA was injected into the neck at P4 and P7, prior to sacrifice at P15. By VESGEN 2D network analysis, TA strongly decreased vessel density and vessel diameter within peripheral vessel networks, compared to PBS-treated control. Decreased vessel density in the TA-treated retina was associated with decreased numbers of considerably enlarged avascular spaces (lacunae). As illustrated by VESGENgenerated vascular maps (DM) and colorized skeleton overlays (Fig. 6B-C, E-F), vessel 
area density $\left(\mathrm{A}_{\mathrm{v}}\right)$ decreased from 0.42 to 0.25 , vessel branch point density $\left(\mathrm{Br}_{\mathrm{v}}\right)$ from 631 per normalized retinal field to 234 , and average vessel diameter $\left(D_{v}\right)$ from 4.39 pixels to 3.89 pixels. Enclosed avascular spaces (indicated in white, Fig. 6C, F) decreased from 250 to 98 , and the avascular area fraction increased from 0.58 to 0.75 .

\section{Stimulation of Lymphatic Networks in Quail CAM by VEGF}

Application of $\mathrm{VEGF}_{165}$ to the quail CAM at mid-development resulted in multimodal effects to the angiogenic vascular tree, as quantified by fractal-based VESGEN 2D analysis (Parsons-Wingerter et al., 2006a). Furthermore, $V_{E G F} 165$ greatly disrupted normal lymphatic architecture, which by E8 is organized as small lymphatic vessels loosely surrounding the smaller blood vessels and elaborated as large lymphatic networks tightly enclosing larger blood vessels (Fig. 7). Lymphatic vessels treated by $\mathrm{VEGF}_{165}$ dissociated from blood vessels, widened considerably and reorganized into a loose network that resembled earlier, less differentiated lymphatic architecture (Fig. 7B). By VESGEN 2D network analysis, vessel diameter and diameter variability increased, as did the area of enclosed avascular spaces. Increased area for the avascular spaces was associated with decreased number density of these spaces. Interestingly, as a fractal rescaling, vessel area density $\left(A_{v}\right)$ for ROIs in Fig. 7D and G remained relatively constant at 0.357 and 0.355 , although the avascular area expanded considerably by a factor of 2.3X. For VEGF-treated lymphatics, average vessel diameter $\left(D_{v}\right)$ increased from 12.4 pixels to 21.6 pixels and average area of avascular spaces, from 4229 pixels to 16687 pixels. Although the lymphatic network in Fig. 7E is a highly planar structure and hence relatively 2D, the large networks and small vessels in Fig. 7A are clearly more 3D. These structures would be analyzed with increased accuracy by a VESGEN 3D analysis, which we plan to develop soon. Although our lymphatic network analysis was expanded using recent, new capabilities of VESGEN $2 \mathrm{D}$, this study of the effects of $\mathrm{VEGF}_{165}$ is described more completely in a previous study (Parsons-Wingerter et al., 2006a).

\section{Vascular Network-to-Tree Transitions in Developing Coronary Vessels}

Vascular trees, which are tapering, asymmetric structures characterized by bifurcational branching, often form from the progressive remodeling of relatively symmetric, homogeneous vascular networks. Embryonic coronary vessels, for example, begin as a vascular network that originates as isolated capillary plexuses forming at approximately embryonic day E12.5 in the mouse (Fig. 8A-C) and Hamburger and Hamilton (HH) Stage 27 (approximately E5.5) in the chick or quail (Sedmera and Watanabe, 2006; Tomanek et al., 2006). Coronary vessels will eventually attach to the aorta at E14.5 in the mouse and at $\mathrm{HH}$ stage 32 in the avian embryo. By E15.5, the undifferentiated murine coronary network has developed into an immature network-tree composite (Fig. 8D-G) that retains network features such as non-tapering vessels and anastomotic vascular loops. As illustrated by $\mathrm{HH}$ Stage 35 in the chicken heart (approximately E9), a more mature vascular tree displays fully tapering vessels labeled positively for smooth muscle cells for several generations of bifurcational branching (Fig. 8H-I).

Coronary vascular architecture begins as very small, undifferentiated networks (plexuses) of capillary vessels (Fig. 8A). Visualization of an initial, small capillary plexus requires much larger magnification (200X) than the low magnification (5X) required for imaging the much larger, more mature vascular tree and its location on the heart (Fig. 8H-I). Mapping and quantification of coronary vessel architecture by VESGEN 2D was used at these different microscopic length scales to characterize the important, fascinating development of coronary vessels from small, undifferentiated (homogeneous or symmetric) capillary networks into a mature, nonhomogeneous (asymmetric), tapering vascular tree. 


\section{DISCUSSION}

Applications of VESGEN 2D software were reviewed for several vascular trees and networks. Operating as an easily installed Java-based plug-in to NIH ImageJ, VESGEN 2D is now fully automated, user-interactive, and includes a variety of useful vascular mapping and quantification options. Experimental and clinical research models of angiogenesis and lymphangiogenesis analyzed by VESGEN 2D included progressive vascular disease in the human retina; multimodal effects of the anti-angiogenic steroid triamcinolone acetonide (TA) on vascular trees of the quail CAM and vascular networks of postnatal mouse retina and of VEGF 165 on CAM lymphangiogenic networks, and network-to-tree transitions of coronary vessels in the mouse and avian embryonic heart. Differences between the human retinal disease examples and between in vivo experimental results were quantified and also illustrated by relevant vascular mapping.

It is clear that the averaging of many important parameters within a vascular tree such as vessel diameter and vessel length is not particularly useful or meaningful when these parameters vary so greatly throughout the vascular tree. VESGEN 2D therefore measures many of these parameters according to site-specific locations within the branching tree. For example, $D_{\mathrm{v} 1-3}$ denotes vessel diameter $\mathrm{D}_{\mathrm{v}}$ with respect to branching generations G1-G3, and $\mathrm{N}_{\mathrm{v} 6}$ is the vessel number density $\mathrm{N}_{\mathrm{v}}$ for branching generation G6. This approach allowed us to conclude that angiogenic regulators such as TA, VEGF 165 , bFGF and TGF- $\beta 1$ stimulated or inhibited angiogenesis within a branching vascular tree only at the level of new, small vessels (Parsons-Wingerter et al., 2000a; Parsons-Wingerter et al., 2000b; Parsons-Wingerter et al., 2006b; McKay et al., 2008) and that VEGF 165 increased vessel diameter only in larger vessels. Thus far in our studies on quantification of angiogenesis and lymphangiogenesis, inhibition or stimulation of vessel growth typically resulted in a maximum change in vessel density or other critical parameters ranging from approximately $1.5 \mathrm{X}$ to $4.0 \mathrm{X}$. We anticipate that the site-specific analysis of regulatory effects by VESGEN 2D will help assess the degree of vascular normalization achieved by new vascular therapeutics.

Using a coupled analysis of binary and skeletonized (linearized) images, VESGEN 2D analyzes complex vascular patterns according to basic connectivity principles of 8-pixel neighborhoods and recent advances in distance mapping (DM or Euclidean distance mapping, EDM)(Russ, 1994; Parsons-Wingerter et al., 2006a). As shown by Kassab and coworkers (Gan et al., 1993; Kassab et al., 1993; Kassab et al., 1994), vessel diameter, not vessel length or branching angle, is the strongest correlate of vessel branching generation in 3D arterial and venous trees of the mammalian heart and lung. Relatively equivalent (symmetric), bifurcational branching as determined by an approximate $71 \%$ decrease in vessel diameter was therefore adopted as the primary basis of vessel segmentation (generational assignment) by VESGEN 2D tree analysis. However, the most frequent branching event within a tapering vascular tree is nonequivalent branching (branching of a smaller vessel from a much larger vessel), not equivalent or symmetric branching (branching of a parent vessel into two equal offspring vessels).

Interestingly, each angiogenesis regulator tested in the quail CAM such as TA, $\mathrm{VEGF}_{165}$, bFGF, TGF- $\beta 1$ or angiostatin has induced a unique 'fingerprint' or 'signature' change in vascular patterning when evaluated by fractal/VESGEN analysis (Parsons-Wingerter et al., 1998; Parsons-Wingerter et al., 2000a; Parsons-Wingerter et al., 2000b; Parsons-Wingerter et al., 2006a; Parsons-Wingerter et al., 2006b; McKay et al., 2008). These results suggest that VESGEN analysis may provide a useful integrative read-out of dominant molecular signaling operating within complex angiogenic programs (Hanahan and Folkman, 1996). The quail CAM has been a convenient experimental and analytical testbed for developing 
new quantification methods. For example, a region-based fractal analysis developed originally in the CAM was successfully applied to quantification of vascular disease progression in the human retina (Avakian et al., 2002). In the future, VESGEN 2D will be extended to 3D vascular trees and networks, and will incorporate bioinformatic and complexity dimensions such as endothelial cell receptor density and localization and multiphase dynamics of blood flow.

\section{Acknowledgments}

We thank Dr. Robert Visconti, Medical University of South Carolina, for reproduction of his confocal image of the mouse coronary vascular network (Fig. 8A). We thank Dr. Steven Fisher for helpful discussion and acknowledge support by NASA IRD04-54, NIH EY017528, EY017529, K12HD049091, HL65314, HL0775436, HL083048, ES013507 and CA92440.

\section{REFERENCES}

Avakian A, Kalina RE, Sage EH, Rambhia AH, Elliott KE, Chuang EL, Clark JI, Hwang J-N, ParsonsWingerter P. Fractal analysis of region-based vascular change in the normal and non-proliferative diabetic retina. Curr Eye Res. 2002; 24:274-280. [PubMed: 12324866]

Barbosky L, Lawrence DK, Karunamuni G, Wikenheiser JC, Doughman YQ, Visconti RP, Burch JB, Watanabe M. Apoptosis in the developing mouse heart. Dev Dyn. 2006; 235:2592-2602. [PubMed: 16881058]

Benjamin LE, Hemo I, Keshet E. A plasticity window for blood vessel remodelling is defined by pericyte coverage of the preformed endothelial network and is regulated by PDGF-B and VEGF. Development. 1998; 125:1591-1598. [PubMed: 9521897]

Brasil OF, Smith SD, Galor A, Lowder CY, Sears JE, Kaiser PK. Predictive factors for short-term visual outcome after intravitreal triamcinolone acetonide injection for diabetic macular oedema: an optical coherence tomography study. Br J Ophthalmol. 2007; 91:761-765. [PubMed: 17108013]

Folkman J. Angiogenesis: an organizing principle for drug discovery? Nat Rev Drug Discov. 2007; 6:273-286. [PubMed: 17396134]

Galor A, Margolis R, Brasil OM, Perez VL, Kaiser PK, Sears JE, Lowder CY, Smith SD. Adverse events after intravitreal triamcinolone in patients with and without uveitis. Ophthalmology. 2007; 114:1912-1918. [PubMed: 17908594]

Gan RZ, Tian Y, Yen RT, Kassab GS. Morphometry of the dog pulmonary venous tree. J. Appl. Physiol. 1993; 75:432-440. [PubMed: 8376295]

Gerhardt H, Golding M, Fruttiger M, Ruhrberg C, Lundkvist A, Abramsson A, Jeltsch M, Mitchell C, Alitalo K, Shima D, Betsholtz C. VEGF guides angiogenic sprouting utilizing endothelial tip cell filopodia. J. Cell Biol. 2003; 161:1163-1177. [PubMed: 12810700]

Hanahan D, Folkman J. Patterns and emerging mechanisms of the angiogenic switch during tumorigenesis. Cell. 1996; 86:353-364. [PubMed: 8756718]

Kassab GS, Lin DH, Fung YC. Morphometry of pig coronary venous system. Am. J. Physiol. 1994; 267:H2100-H2113. [PubMed: 7810711]

Kassab GS, Rider CA, Tang NJ, Fung YC. Morphometry of pig coronary arterial trees. Am. J. Physiol. 1993; 265:H350-H365. [PubMed: 8342652]

LaRue AC, Mironov VA, Argraves WS, Czirok A, Fleming PA, Drake CJ. Patterning of embryonic blood vessels. Dev Dyn. 2003; 228:21-29. [PubMed: 12950076]

Lazarovici P, Gazit A, Staniszewska I, Marcinkiewicz C, Lelkes PI. Nerve growth factor (NGF) promotes angiogenesis in the quail chorioallantoic membrane. Endothelium. 2006; 13:51-59. [PubMed: 16885067]

Mandelbrot, BB. The Fractal Geometry of Nature. San Francisco: W. H. Freeman; 1983.

McKay TL, Gedeon DJ, Vickerman MB, Hylton AG, Ribita D, Olar HH, Kaiser PK, ParsonsWingerter P. Selective inhibition of angiogenesis in small blood vessels and decrease in vessel diameter throughout the vascular tree by triamcinolone acetonide. Invest Ophthalmol Vis Sci. 2008; 49:1184-1190. [PubMed: 18326748] 
Moshfeghi DM, Kaiser PK, Bakri SJ, Kaiser RS, Maturi RK, Sears JE, Scott IU, Belmont J, Beer PM, Quiroz-Mercado H, Mieler WF. Presumed sterile endophthalmitis following intravitreal triamcinolone acetonide injection. Ophthalmic Surg Lasers Imaging. 2005; 36:24-29. [PubMed: 15688968]

Parsons-Wingerter P, Chandrasekharan UM, McKay TL, Radhakrishnan K, DiCorleto PE, Albarran B, Farr AG. A VEGF165-induced phenotypic switch from increased vessel density to increased vessel diameter and increased endothelial NOS activity. Microvasc Res. 2006b; 72:91-100. [PubMed: 16872639]

Parsons-Wingerter P, Elliott KE, Clark JI, Farr AG. Fibroblast growth factor-2 selectively stimulates angiogenesis of small vessels in arterial tree. Arterioscler. Thromb. Vasc Biol. 2000b; 20:12501256. [PubMed: 10807740]

Parsons-Wingerter P, Elliott KE, Farr AG, Radhakrishnan K, Clark JI, Sage EH. Generational analysis reveals that TGF-beta1 inhibits the rate of angiogenesis in vivo by selective decrease in the number of new vessels. Microvasc. Res. 2000a; 59:221-232. [PubMed: 10684728]

Parsons-Wingerter P, Lwai B, Yang MC, Elliott KE, Milaninia A, Redlitz A, Clark JI, Sage EH. A novel assay of angiogenesis in the quail chorioallantoic membrane: stimulation by bFGF and inhibition by angiostatin according to fractal dimension and grid intersection. Microvasc. Res. 1998; 55:201-214. [PubMed: 9657920]

Parsons-Wingerter P, McKay TL, Leontiev D, Vickerman MB, Condrich TK, Dicorleto PE. Lymphangiogenesis by blind-ended vessel sprouting is concurrent with hemangiogenesis by vascular splitting. Anat Rec A Discov Mol Cell Evol Biol. 2006a; 288:233-247. [PubMed: 16489601]

Russ, JC. The Image Processing Handbook. 2nd ed.. Boca Raton, FL: CRC Press, Inc; 1994.

Sedmera D, Watanabe M. Growing the coronary tree: the quail saga. Anat Rec A Discov Mol Cell Evol Biol. 2006; 288:933-935. [PubMed: 16906547]

Tomanek RJ, Hansen HK, Dedkov EI. Vascular patterning of the quail coronary system during development. Anat Rec A Discov Mol Cell Evol Biol. 2006; 288:989-999. [PubMed: 16892426] 

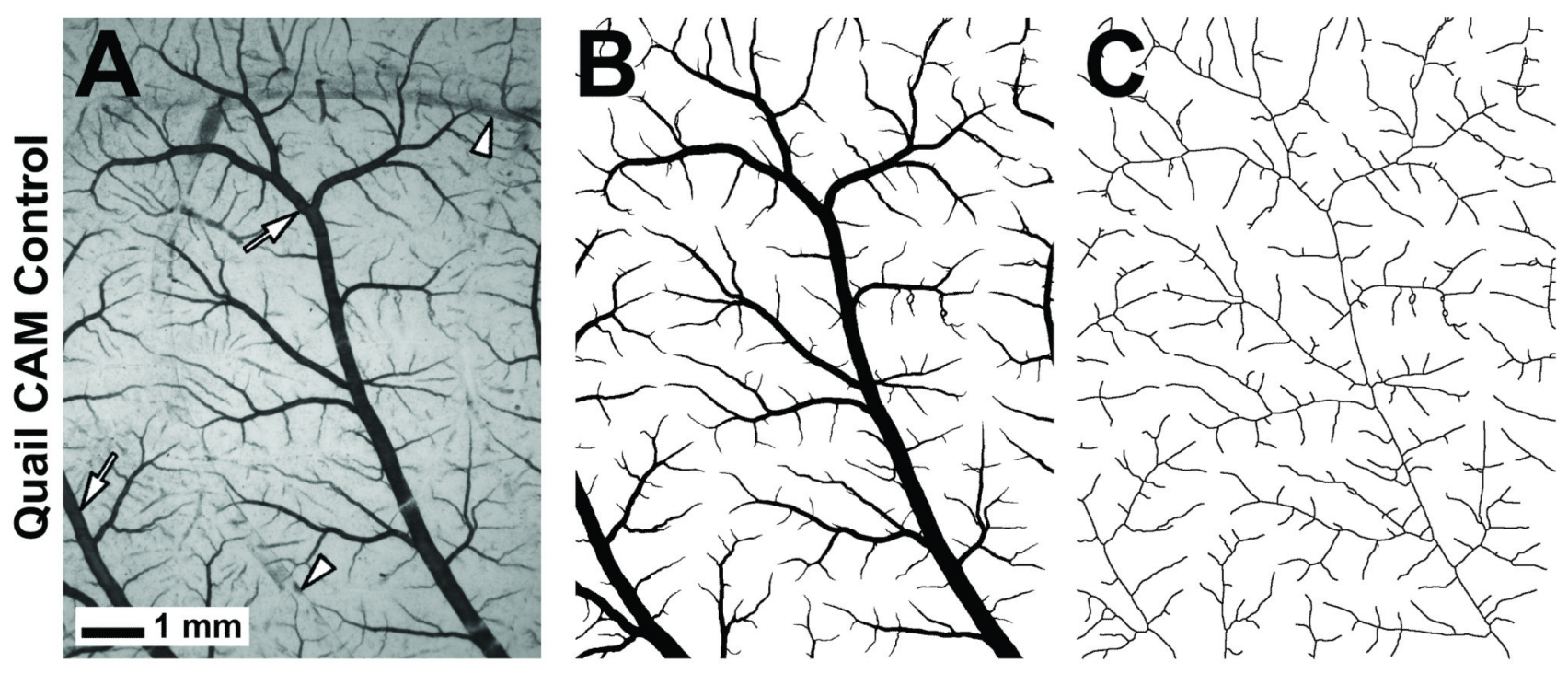

\section{VESGEN Tree Output}

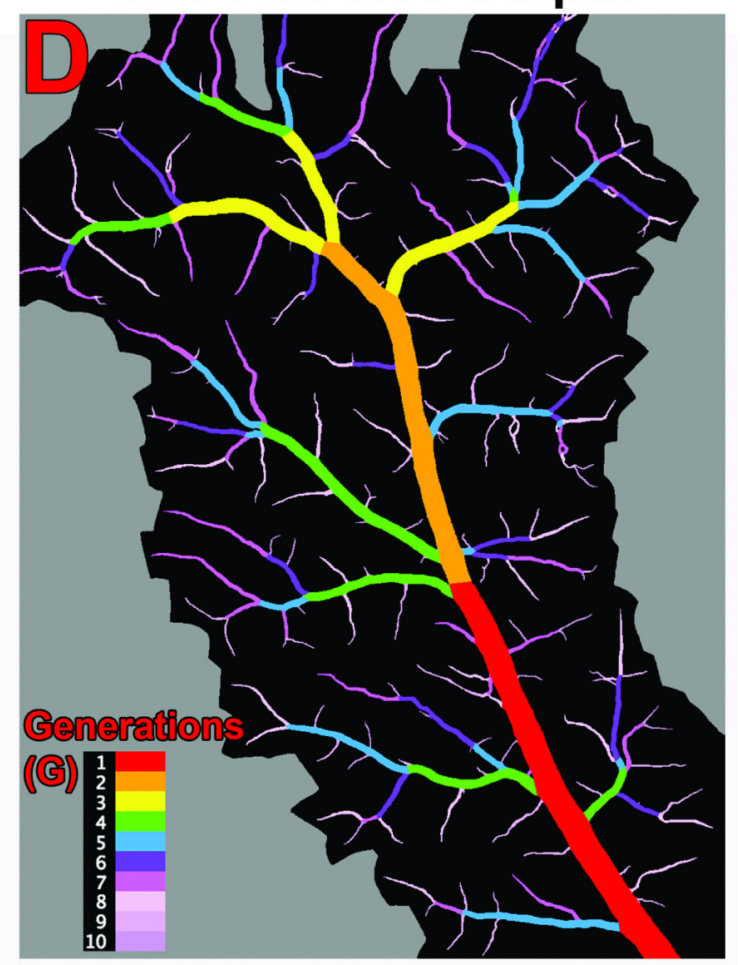

Fig. 1. VESGEN 2D Analysis of Branching Vascular Trees

(A) To obtain binary vascular images for VESGEN 2D analysis in the CAM, the arterial end-point region is generally imaged in grayscale by brightfield microscopy at low magnification (here, $12.5 \times$ total) to maximize the field of vascular branching. Magnification was also selected to exclude the CAM capillary bed (typically visualized at $\geq 20 \times$ total), because the capillaries are organized as a symmetric, homogeneous vascular network, and are thus morphologically distinct from the branching arterial and venous trees. The E8 CAM specimen illustrates how aldehyde fixation results in the retention of blood containing red blood cells (RBCs) within arteries (arrows), but not in veins (arrowheads). Arterial trees are thereby conveniently separated from overlapping venous trees, and (B) extracted by semi- 
automatic computer processing as a binary (black/white) vascular pattern. (C) VESGEN 2D generates the skeletonized (linearized) image of vascular connectivity from (B), the single input binary image. Vessel skeletons in this and all other figures have been widened for illustration (by definition, skeletons are one pixel in width). The software then maps and quantifies the vascular tree using various image processing algorithms to analyze these two geometric representations (B, C). (D) This VESGEN 2D output displays the major arterial tree with its automatically determined ROI (in black) and classification of vessels into ten successively smaller branching generations, $\mathrm{G}_{1}-\mathrm{G}_{10}$. For this $\mathrm{E} 8$ control specimen, for example, $(\mathbf{B})$ vessel area density $\left(\mathrm{A}_{\mathrm{v}}\right)=0.136\left(\mathrm{~cm}^{2} / \mathrm{cm}^{2}\right)$ and fractal dimension $\left(\mathrm{D}_{\mathrm{f}}\right)=1.66$; (C) vessel length density $\left(\mathrm{L}_{\mathrm{v}}\right)=25.2\left(\mathrm{~cm} / \mathrm{cm}^{2}\right)$, vessel branch-point density $\left(\mathrm{Br}_{\mathrm{v}}\right)=380$ $\left(\mathrm{cm}^{-2}\right)$ and $\mathrm{D}_{\mathrm{f}}=1.40$. For the extracted major vascular tree $(\mathbf{D}), \mathrm{A}_{\mathrm{v} 1-3}=0.057\left(\mathrm{~cm}^{2} / \mathrm{cm}^{2}\right)$, $\mathrm{L}_{\mathrm{v} 1-3}=2.8\left(\mathrm{~cm} / \mathrm{cm}^{2}\right)$, and $\mathrm{N}_{\mathrm{v} 1-3}=11.4\left(\mathrm{~cm}^{-2}\right)$, compared to $\mathrm{A}_{\mathrm{v} 7-10}=0.040\left(\mathrm{~cm}^{2} / \mathrm{cm}^{2}\right)$, $\mathrm{L}_{\mathrm{v} 7-10}=15.1\left(\mathrm{~cm} / \mathrm{cm}^{2}\right)$, and $\mathrm{N}_{\mathrm{v} 7-10}=397.3\left(\mathrm{~cm}^{-2}\right)$. 

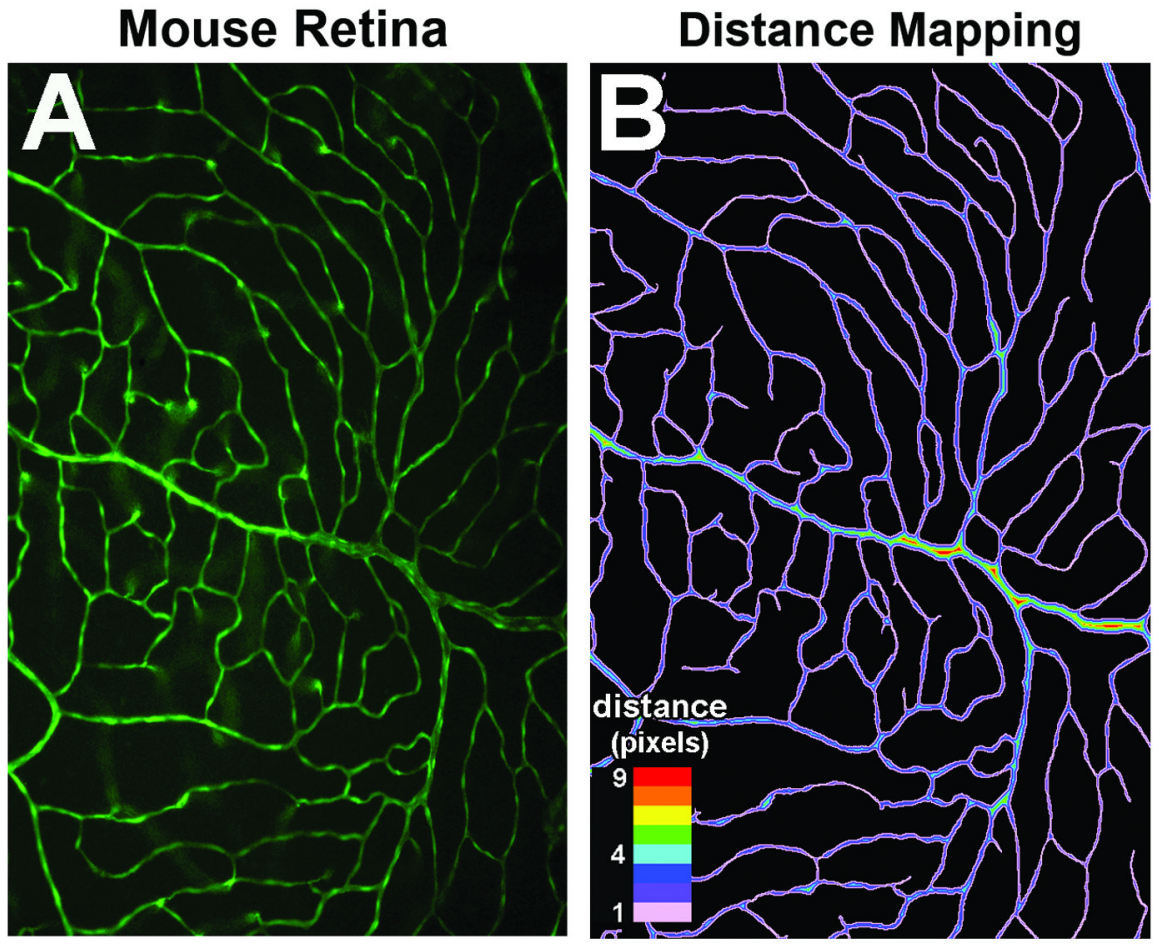

VESGEN Network Output Colorized Skeleton

Fig. 2. VESGEN 2D Analysis of Vascular Networks

Obtaining a VESGEN 2D analysis of a vasculogenic or mature capillary network is similar to the previous example of a branching arterial tree (Fig. 1). (A) The vasculogenic capillary network from a P15 postnatal mouse retina visualized by Tie2/GFP expression at $10 \times$ total magnification was binarized and for this example, $(\mathbf{B})$ a Euclidean distance map (EDM or DM) was generated from the binary pattern by VESGEN 2D. As indicated by the look-up table (LUT), colors within the vascular DM correspond to pixel distance to edge of vessel. For this vascular network, VESGEN 2D measured the vessel area density $\left(A_{V}\right)$ as 0.14 , vessel branch point density $\left(\mathrm{Br}_{\mathrm{v}}\right)$ of 164 per retinal field, average vessel diameter $\left(\mathrm{D}_{\mathrm{V}}\right)$ of 4.83 pixels, 50 enclosed avascular spaces, and avascular area fraction of 0.86 . (C) By another vascular mapping option, the colorized skeleton was overlaid on the binary pattern where colors again correspond to thickness (or radius) of the vessel at the location of each skeletal point as given by the LUT $(\mathbf{B})$. Averaging DM values at points along the skeleton can be used to estimate the average radius of local vessel segments as well as of the entire vessel network. 

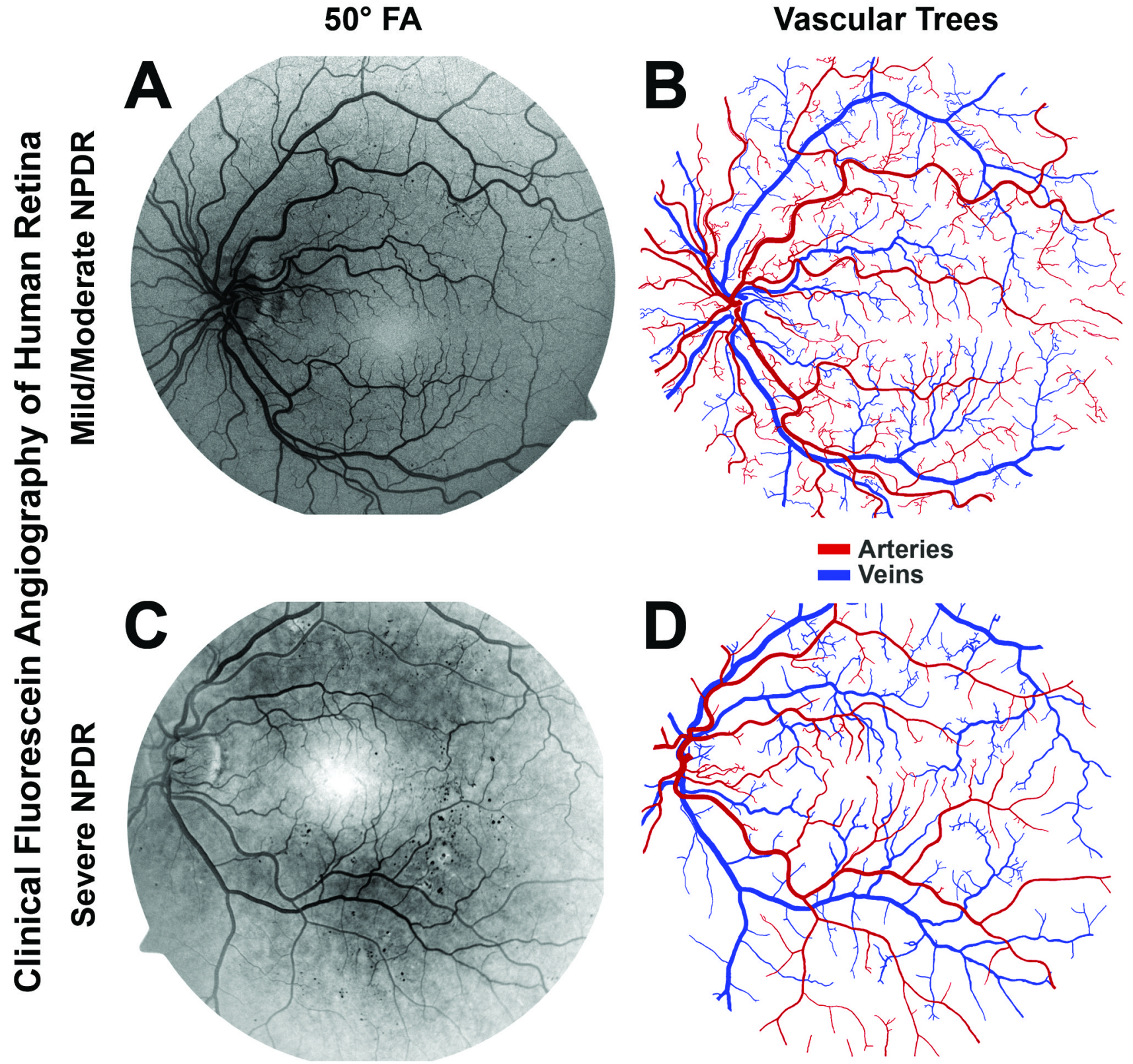

Fig. 3. Extraction of Vascular Trees from Clinical Images of the Human Retina

Vascular trees were extracted from $(\mathbf{A}, \mathbf{C})$ grayscale ophthalmic images obtained by clinical FA using semi-automatic computer processing (B, D). Arterial and venous trees were distinguished from each other according to FA guidelines for retinal arterio-venous anatomy, fluorescein filling, and vessel connectivity, as well as well-established morphological characteristics such as the smaller diameters and increased vessel tortuosity of arteries compared to veins. (B, D) The appearance of the overlapping arterial and venous trees suggests that significant vessel dropout occurred during the progression from mild/ moderate (A) to severe (C) nonproliferative diabetic retinopathy (NPDR) as diagnosed clinically using other (secondary) vascular markers such as microaneurysm density. 
Arterial Tree
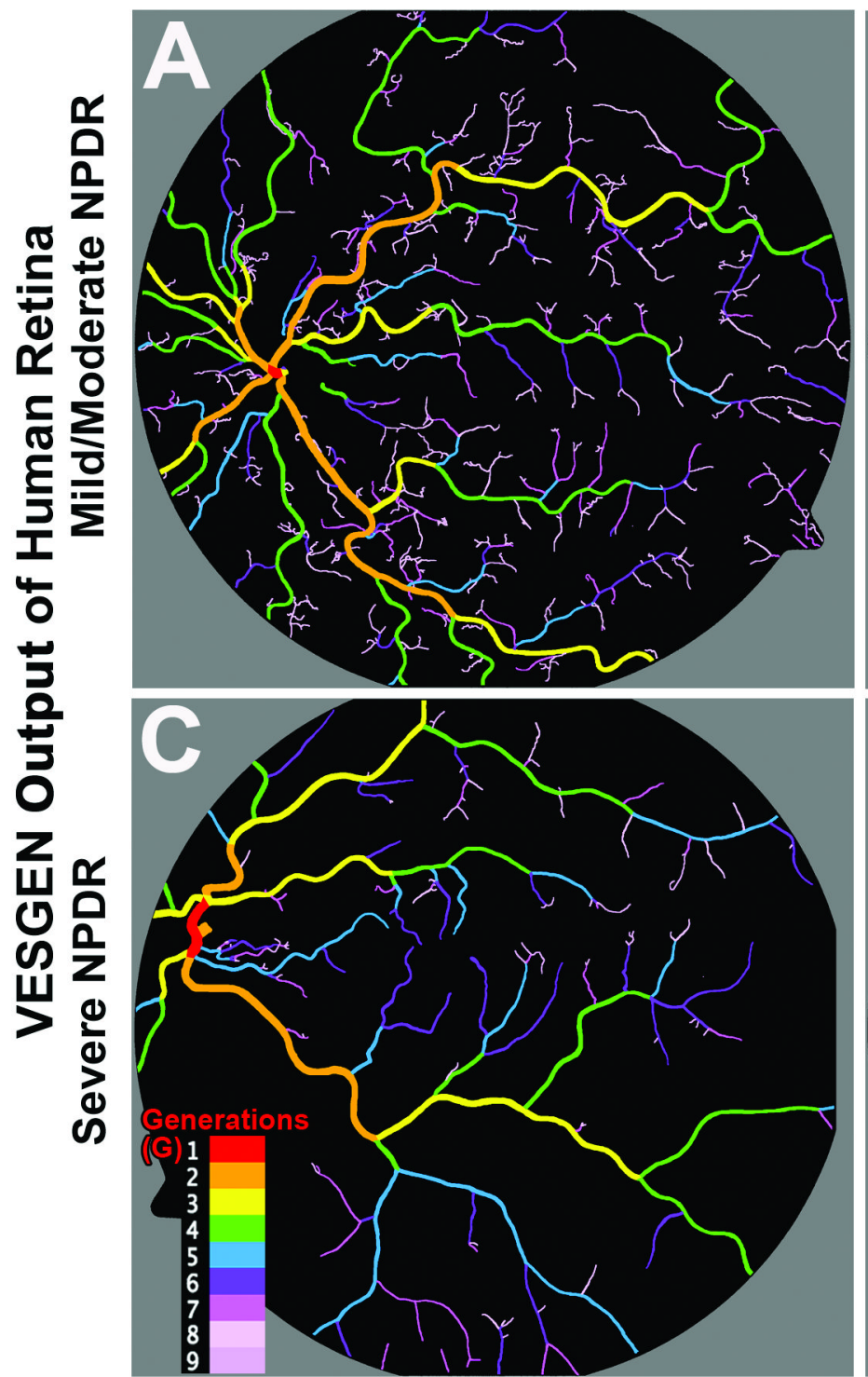

Venous Tree
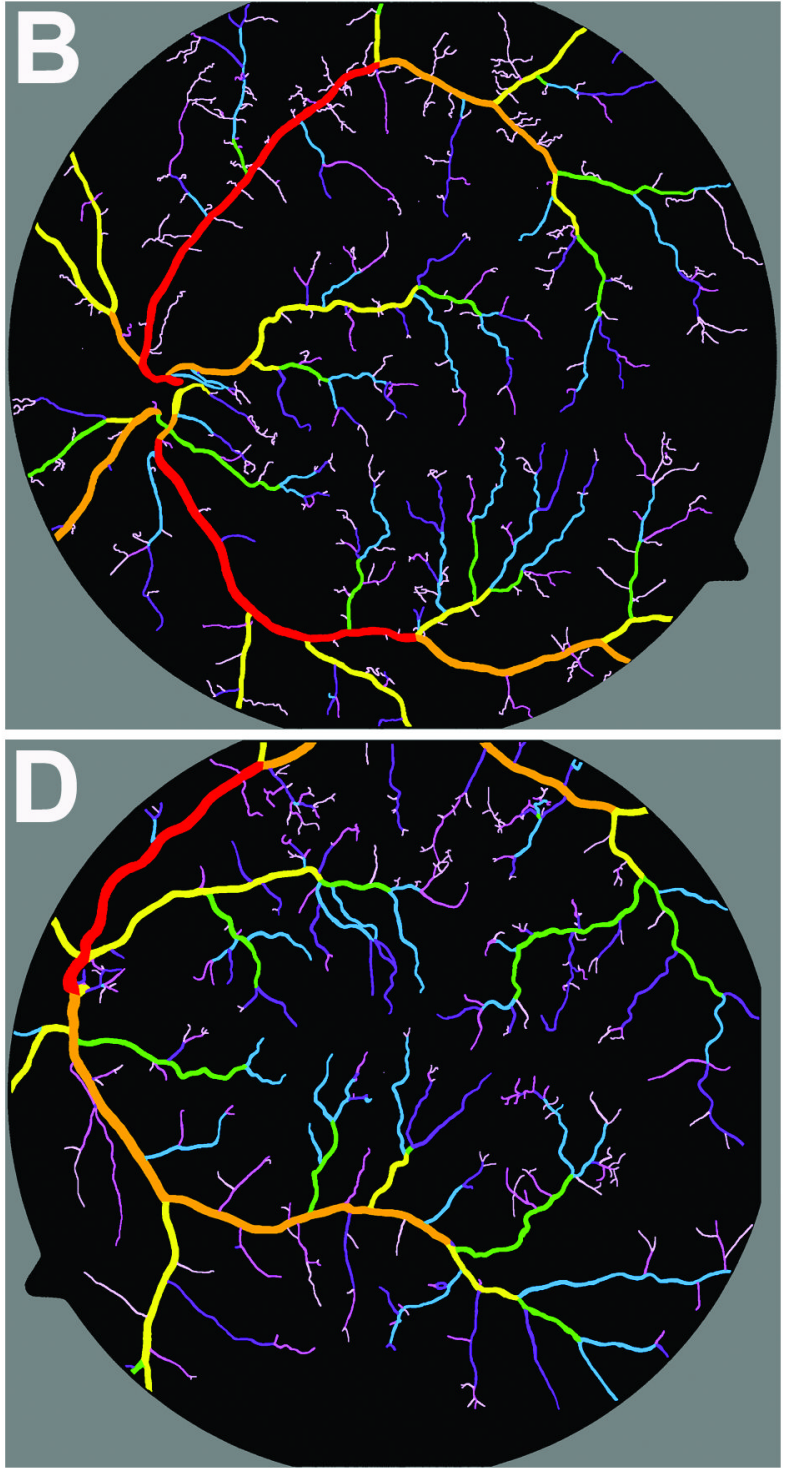

Fig. 4. VESGEN 2D Analysis of NPDR Progression in the Human Retina

By VESGEN 2D measurements in generation-specific maps of $(\mathbf{A}, \mathbf{C})$ arterial trees and $(\mathbf{B}$, D) venous trees, the largest difference between the mild/moderate NPDR and severe NPDR images was a large decrease in the number and density of smaller arteries (G5-G9), compared to decrease in the density of larger arteries (G1-G4). For example, vessel number density $\mathrm{N}_{\mathrm{v} 5-9}$ decreased by $76 \%$ (from 629 arteries per normalized retinal field to 155), and $\mathrm{N}_{\mathrm{v} 1-4}$, by $44 \%$ (from 36 arteries per retinal field to 20 ) in the severe NPDR arterial tree (C) relative to mild/moderate NPDR (A). Throughout the entire arterial trees of $(\mathbf{C})$ relative to (A), vessel area density $\left(\mathrm{A}_{\mathrm{v} 1-9}\right)$ decreased by $30 \%$, vessel length density $\left(\mathrm{L}_{\mathrm{v} 1-9}\right)$ by $51 \%$, and vessel branch-point density $\left(\mathrm{Br}_{\mathrm{v} 1-9}\right)$ by $78 \%$. $\mathrm{Br}_{\mathrm{v}}$ typically correlates closely with $\mathrm{N}_{\mathrm{v}}$. Differences between the numbers and density of venous vessels were much smaller. Within the entire venous trees of (D) relative to (B), $\mathrm{A}_{\mathrm{v} 1-9}$ increased by $8 \%$; $\mathrm{L}_{\mathrm{v} 1-9}$ and $\mathrm{Br}_{\mathrm{v} 1-9}$ decreased by $15 \%$ and $30 \%$. Decreased arterial density in the severe NPDR image could result from several causes, including arterial oblation, and/or a decrease in arterial diameter below the limit of imaging resolution, which is approximately $40 \mu \mathrm{m}$. 

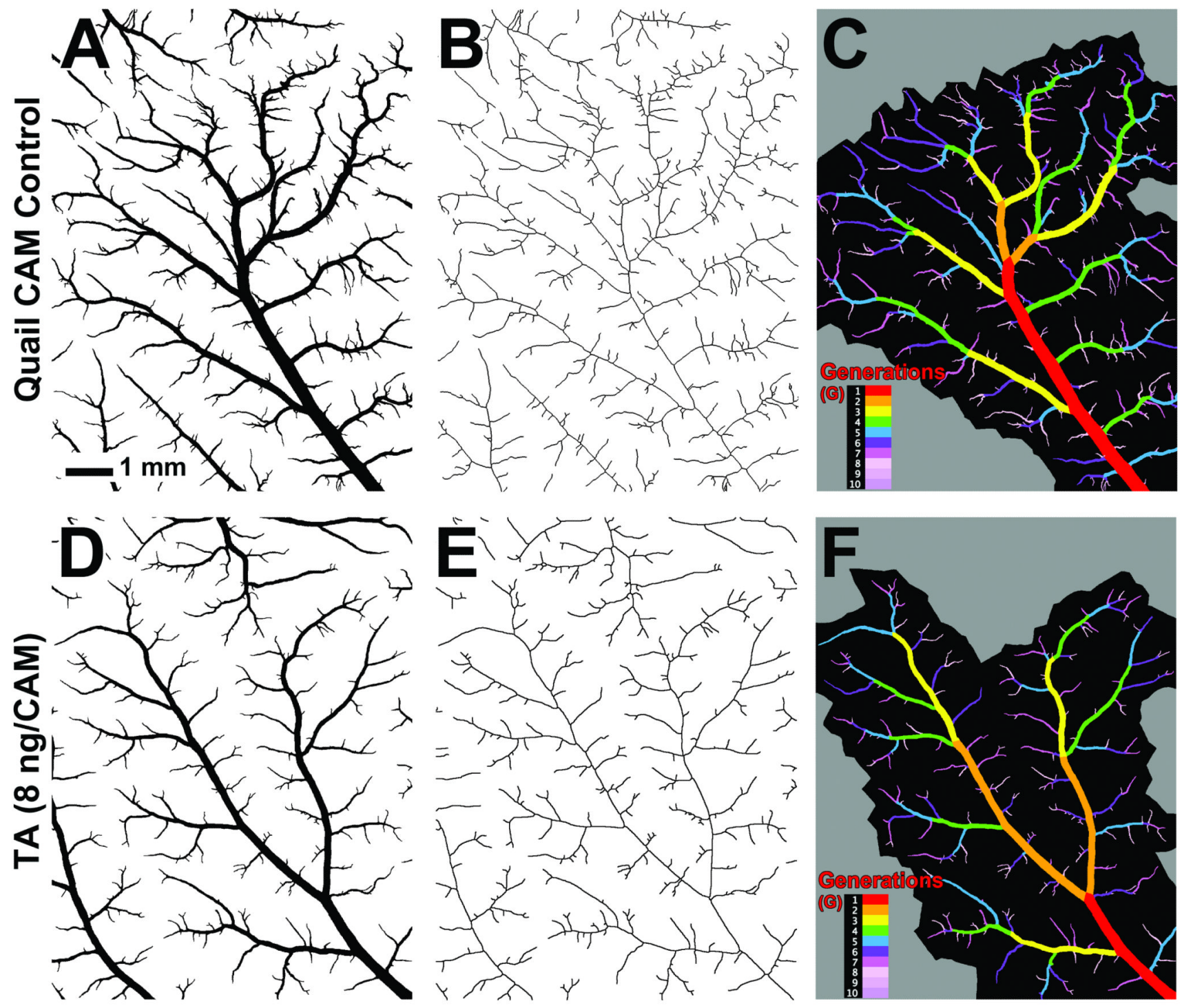

Fig. 5. In the CAM, Anti-Angiogenic Regulation by TA Decreases Selectively the Number of Smaller Vessels, but Decreases Vessel Diameter throughout the Vascular Tree

Binary and skeletonized images of a representative E8 control $(\mathbf{A}, \mathbf{B})$ and TA-treated CAM (D, E) suggest that application of TA decreased both vessel density and vessel diameter. (C, F) Quantification by VESGEN 2D demonstrated TA altered vascular architecture by two major morphological mechanisms: (1) vessel diameters decreased throughout the branching vascular tree, accompanied by (2) selective decrease in the density of smaller blood vessels as indicated by vascular maps of extracted major arterial trees within the ROIs (in black). 


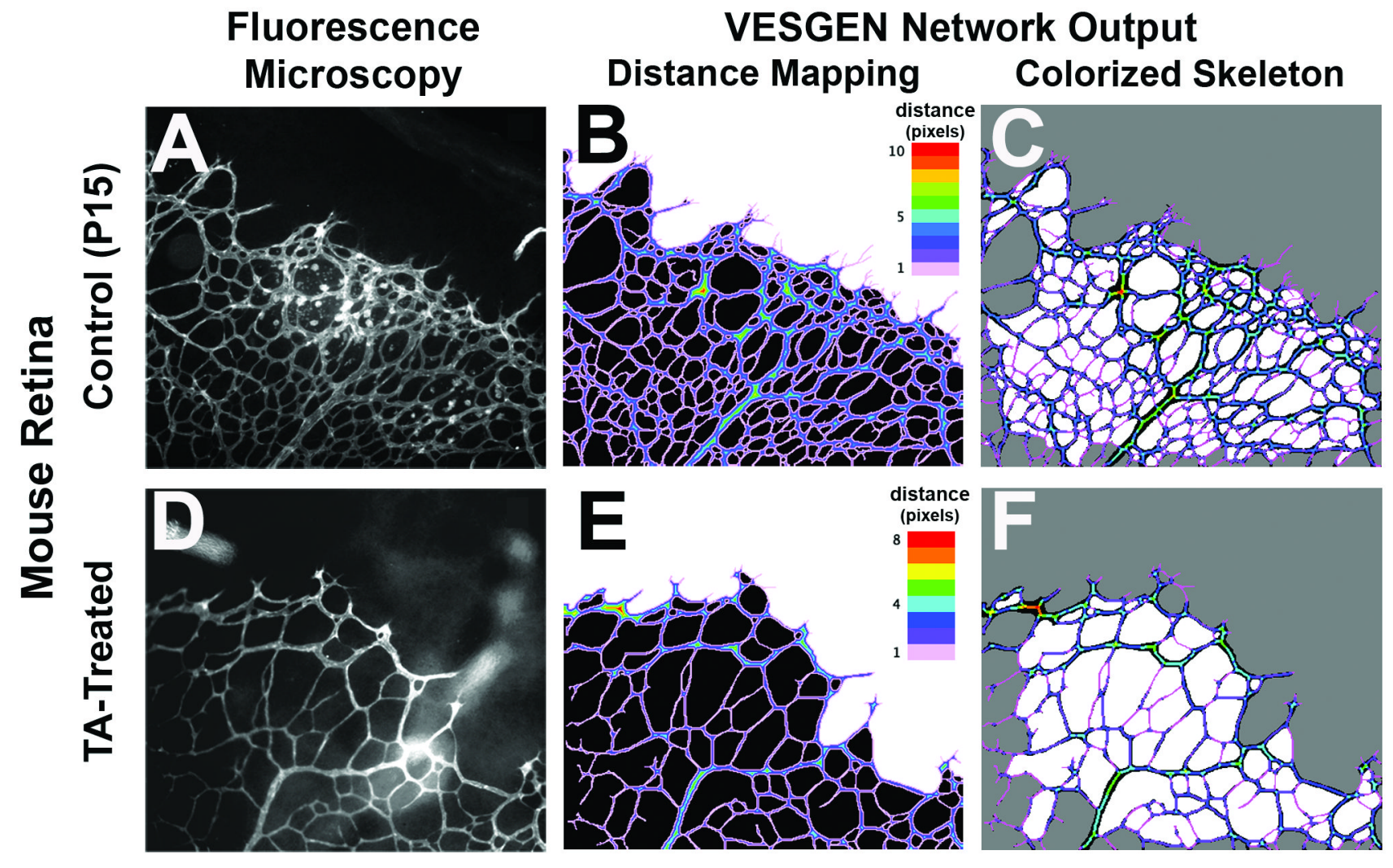

Fig. 6. The Anti-Angiogenic Steroid TA Decreases Vessel Density and Enlarges Avascular Spaces within Vasculogenic Capillary Networks of the Developing Mouse Retina

Compared to PBS-treated control (A-C), vessel density and diameter decreased considerably in the peripheral vasculogenic capillary networks of the developing mouse retina (D-F) following treatment with TA prior to P15. As a consequence, the number of avascular spaces also decreased. Network ROIs are labeled in black (B, E). However, only the numbers and areas of closed avascular spaces (illustrated in white, C, F) are quantified by VESGEN 2D (unclosed avascular spaces, appearing in gray, are excluded). Retinal vasculature was labeled by perfusion of lectin-conjugated fluorescein injected into the heart before sacrifice and after fixation, dissection and mounting, was imaged by fluorescence microscopy at $10 \times$ total magnification. 


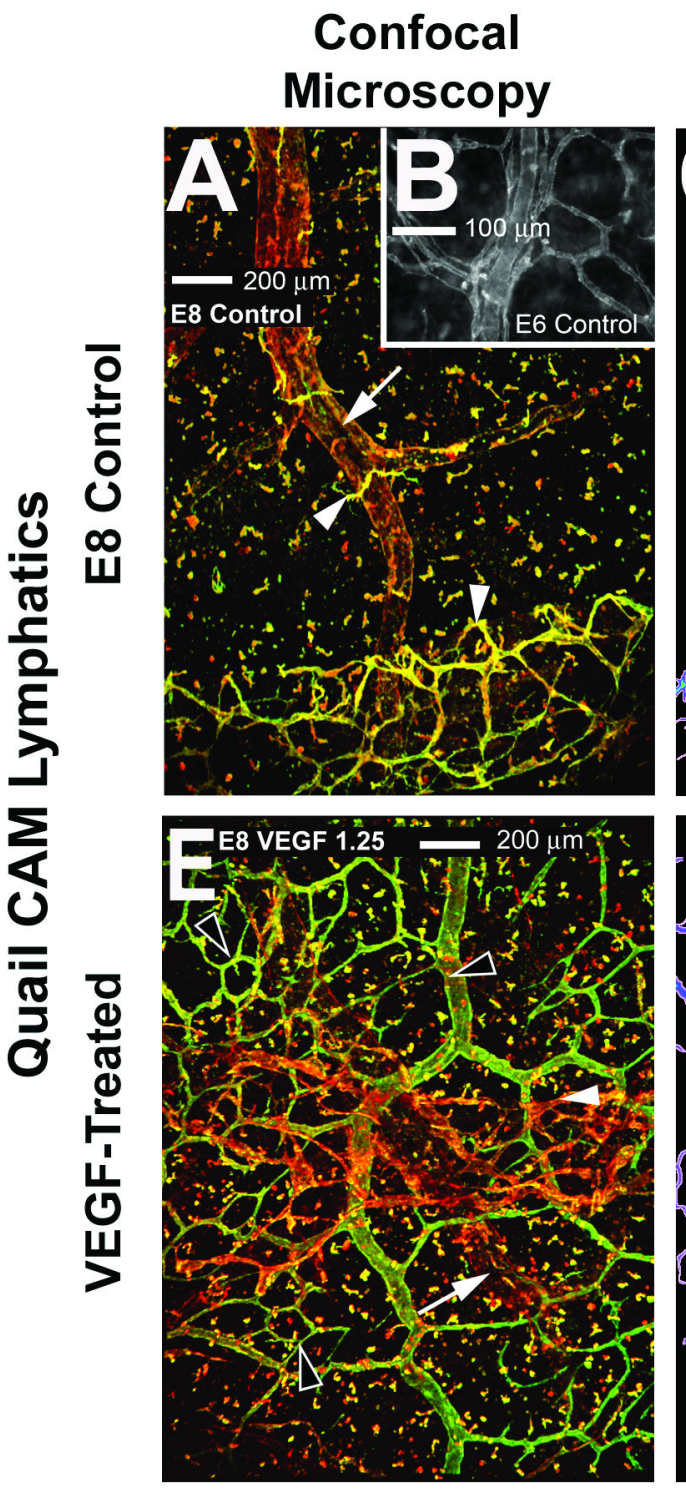

\section{VESGEN Network Output Distance Mapping Colorized Skeleton}
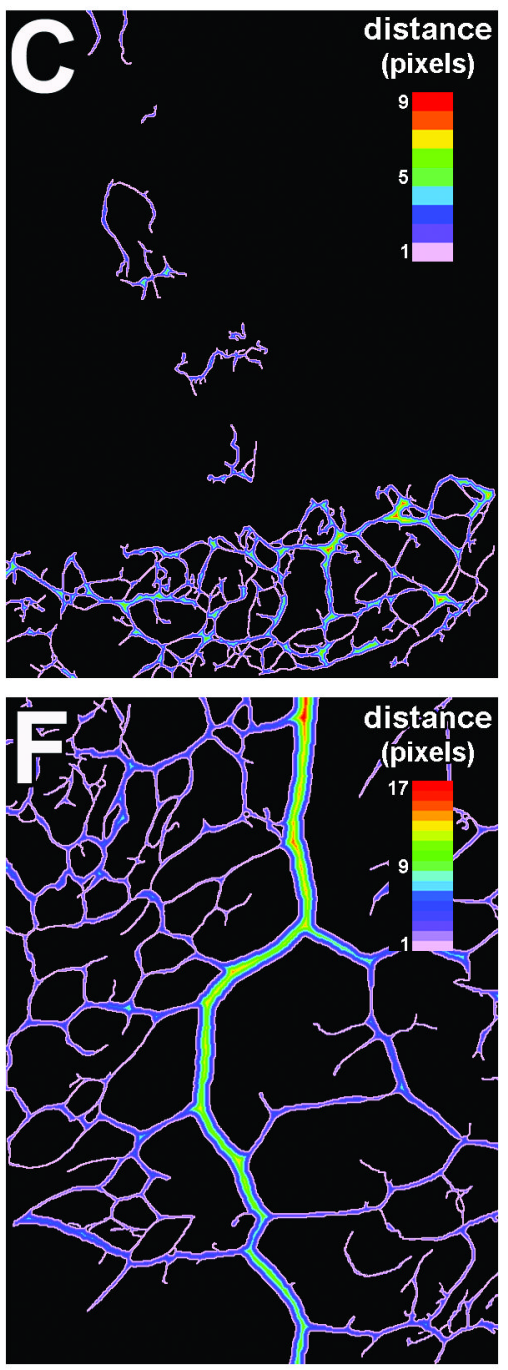
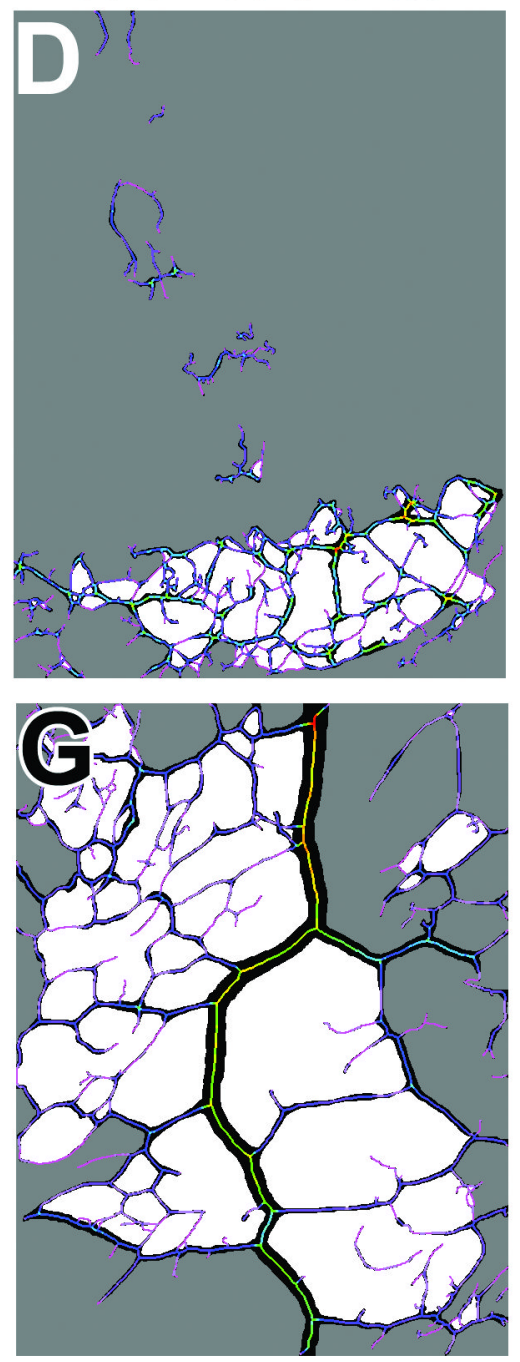

Fig. 7. VEGF 165 Disorganizes Lymphatic Architecture and Enlarges Vessel Diameter and Avascular Spaces of Lymphatic Networks in the CAM

$(\mathbf{A}, \mathbf{E})$ Compared to E8 control, vessel diameter increased-although with large variability-in lymphatic networks of the quail CAM following treatment with $\mathrm{VEGF}_{165}$ for $24 \mathrm{hrs}$. CAM specimens were labeled with antibodies identifying VEGFR-2 (green) and a quail hematopoietic epitope (red). Arrows point to blood vessels, arrowheads to lymphatic vessels and networks, and hollow arrowheads (E) to a large, more homogeneous lymphatic network not associated with blood vessels that is typical of earlier stages of lymphatic development (B). As visualized by confocal microscopy at relatively low magnification, large numbers of isolated vascular progenitor cells appear as punctate staining and are being recruited to growing lymphatics (Parsons-Wingerter et al., 2006a). As shown by (C, F) vascular distance maps $(\mathrm{DM})$ and $(\mathbf{D}, \mathbf{G})$ colorized skeleton overlays generated by VESGEN $2 \mathrm{D}$ network analysis, normal lymphatic architecture consists of highly organized lymphatic networks surrounding large blood vessels and smaller lymphatic vessels encircling smaller blood vessels. The total area of an image, as indicated by large non-lymphatic regions of the CAM ( $\mathbf{C}$ and $\mathbf{F}$, black) is used to quantify lymphatic vessel density and other density parameters 
according to standard practices for normalizing vascular quantities by tissue area. In contrast, white areas $(\mathbf{C}$ and $\mathbf{F})$ indicate the avascular 'island' spaces which are totally enclosed within (compared to the areas of gray avascular spaces which extend beyond the field of the microscopic image); these avascular 'island' spaces are useful for characterizing vascular/avascular relationships within the network (LaRue et al., 2003). (A, B \& E) reprinted with permission of Anatomical Record A. 

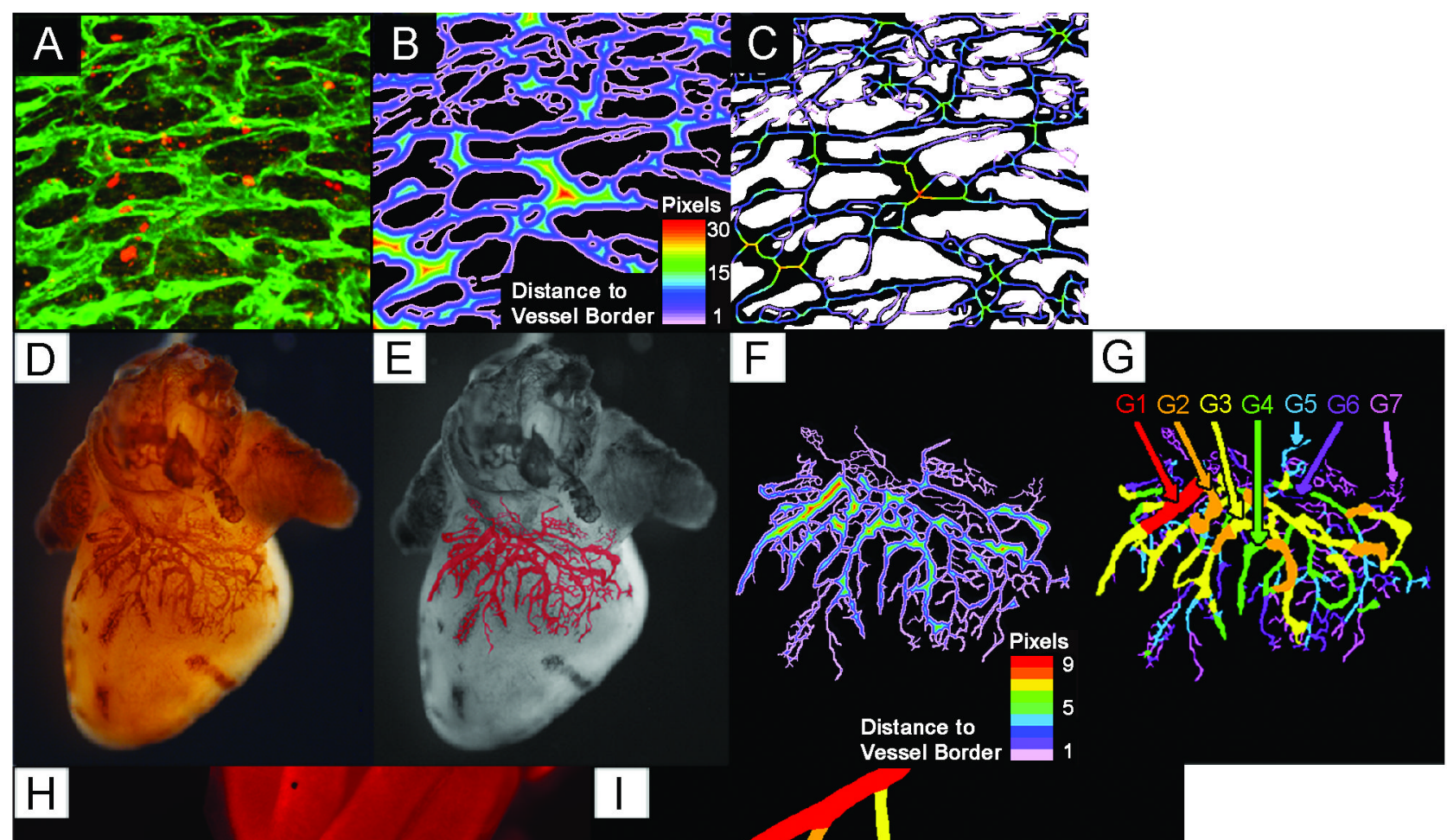

Fig. 8. Development of Embryonic Coronary Vessels from an Immature Vasculogenic Network into a Mature Vascular Tree

(A-C) The coronary vasculature begins as an immature, undifferentiated vasculogenic network when the embryonic heart first grows large enough to require metabolic support by its own blood supply. This coronary network is from an E13.5 mouse heart in which the vessels were labeled by FITC-conjugated CD-31 and imaged by confocal microscopy at $400 \times$. As in previous examples, the coronary network is represented by its DM and a colorized skeleton indicating vessel thickness overlaid upon the binarized network. (D-G) By E15.5, the murine coronary network has remodeled into a vascular network-tree composite (as visualized by brightfield stereo microscopy at low magnification, approximately $9 \times$ ). According to VESGEN 2D tree analysis, branching generations are not yet well defined by successive, orderly, tapering decreases in vessel diameter. The vascular composite contains both tapering branches and anastomatic loops that are characteristic features of trees and networks, respectively. (H-I) By HH35 (approximately E9) in the chicken heart, which matures more quickly than the mouse heart, the coronary vasculature invested by smooth muscle has developed into a relatively mature tree composed of continuously tapering branches. Coronary vessels were labeled by Cy3-conjugated alpha smooth muscle actin ( $\alpha \mathrm{SMA})$ and imaged by fluorescence stereo microscopy at $5 \times$. The 
absence of smaller vessels within this tree results primarily from labeling by $\alpha$ SMA, which is not expressed abundantly on most smaller vessels, the low magnification, and the maturational investment of smaller blood vessels beneath the epicardium. The image in (A) is reproduced with permission from (Barbosky et al., 2006). 